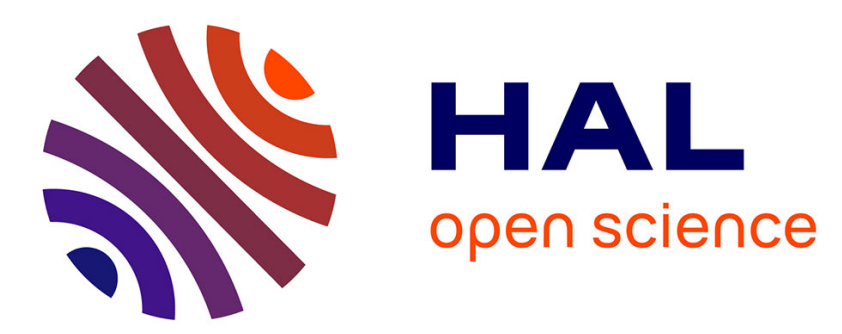

\title{
Les conséquences spatiales de l'intervention de la puissance publique dans l'industrie aérospatiale en Allemagne
}

Denis Eckert

\section{- To cite this version:}

Denis Eckert. Les conséquences spatiales de l'intervention de la puissance publique dans l'industrie aérospatiale en Allemagne. Espace Géographique, 1991, pp.316 - 324. 10.3406/spgeo.1990.3013 . halshs-02565680

\author{
HAL Id: halshs-02565680 \\ https://shs.hal.science/halshs-02565680
}

Submitted on 6 May 2020

HAL is a multi-disciplinary open access archive for the deposit and dissemination of scientific research documents, whether they are published or not. The documents may come from teaching and research institutions in France or abroad, or from public or private research centers.
L'archive ouverte pluridisciplinaire HAL, est destinée au dépôt et à la diffusion de documents scientifiques de niveau recherche, publiés ou non, émanant des établissements d'enseignement et de recherche français ou étrangers, des laboratoires publics ou privés. 


\section{Les conséquences spatiales de l'intervention de la puissance} publique dans l'industrie aérospatiale en Allemagne

Denis Eckert

\section{Citer ce document / Cite this document :}

Eckert Denis. Les conséquences spatiales de l'intervention de la puissance publique dans l'industrie aérospatiale en Allemagne. In: Espace géographique, tome 19-20, n4, 1990. pp. 316-324;

doi : https://doi.org/10.3406/spgeo.1990.3013

https://www.persee.fr/doc/spgeo_0046-2497_1990_num_19_4_3013

Fichier pdf généré le 03/01/2019 


\title{
Zusammenfassung
}

Die räumlichen Folgen des Einsatzes der ôffentlichen Hand in der deutschen Luftfahrtindustrie. Gegen Ende der siebziger Jahre entbrannte in der Bundesrepublik ein heftiger Streit zwischen einigen Nordlândern und dem Bundestaat in bezug auf die Umstrukturierung der Luft und Raumfahrtindustrie. Vom Standpunkt der Regionalbehôrden aus ist die Erhaltung eines technologischen Pols fur eine erfolgreiche Bekàmpfung der Wirtschaftskrise unentbehrlich. Durch diesen Konflikt wird das Eingreifen derôffentlichen Hand auf dem industriellen Gebiet deutlich gemacht.

\begin{abstract}
The spatial consequences of public authority intervention into Germany's aerospace industry. - In the late 1970s a conflict broke out between some of Germany's northern Lander and the Federal State over the restructuring of the aerospace industry. According to the regional authorities it was indispensable to maintain a technological pole in aeronautics in order to face the crisis with some chance of success. This conflict clearly shows the intervention of public authorities in the industrial sector as well as its consequences on space.
\end{abstract}

\section{Résumé}

À la fin des années 1970 en Allemagne fédérale, un conflit aigu éclate entre certains Lander du Nord et l'État fédéral à propos de la restructuration de l'industrie aérospatiale. Dans l'optique des autorités régionales, le maintien d'un pôle technologique aéronautique est indispensable au succès de la lutte contre la crise. Ce conflit met en lumière l'intervention des pouvoirs publics dans le domaine industriel et ses importantes conséquences spatiales. 


\title{
Les conséquences spatiales de l'intervention de la puissance publique dans l'industrie aérospatiale en Allemagne
}

\author{
Denis Eckert
}

CNRS, GIP RECLUS, Montpellier

RÉSUMÉ.- À la fin des années 1970 en Allemagne fédérale, un conflit aigu éclate entre certains Länder du Nord et l'État fédéral à propos de la restructuration de l'industrie aérospatiale. Dans l'optique des autorités régionales, le maintien d'un pôle technologique aéronautique est indispensable au succès de la lutte contre la crise. Ce conflit met en lumière l'intervention des pouvoirs publics dans le domaine industriel et ses importantes conséquences spatiales.

ALLEMAGNE, AMÉNAGEMENT DU TERRITOIRE, DÉVELOPPEMENT RÉGIONAL, INDUSTRIE AÉROSPATIALE

ABSTRACT. - The spatial consequences of public authority intervention into Germany's aerospace industry. - In the late 1970s a conflict broke out between some of Germany's northern Länder and the Federal State over the restructuring of the aerospace industry. According to the regional authorities it was indispensable to maintain a technological pole in aeronautics in order to face the crisis with some chance of success. This conflict clearly shows the intervention of public authorities in the industrial sector as well as its consequences on space.

AIRCRAFT INDUSTRY, GERMANY, LAND PLANNING, REgIONAL DEVELOPMENT,

ZUSAMMENFASSUNG.- Die räumlichen Folgen des Einsatzes der öffentlichen Hand in der deutschen Luftfahrtindustrie.- Gegen Ende der siebziger Jahre entbrannte in der Bundesrepublik ein heftiger Streit $z$ wischen einigen Nordländern und dem Bundestaat in bezug auf die Umstrukturierung der Luftund Raumfahrtindustrie. Vom Standpunkt der Regionalbehörden aus ist die Erhaltung eines technologischen Pols für eine erfolgreiche Bekämpfung der Wirtschaftskrise unentbehrlich. Durch diesen Konflikt wird das Eingreifen deröffentlichen Hand auf dem industriellen Gebiet deutlich gemacht.

DEUTSCHLAND, LUFTFAHRTINDUSTRIE, RAUMORDNUNGSPOLITIK, REGIONALENTWICKLUNG 
L'industrie aérospatiale se présente comme un secteur d'activité assez particulier dans l'économie des pays industrialisés. L'État, par les commandes militaires, en est partout le premier client, sinon le propriétaire des entreprises. Au-delà de son intérêt stratégique, cette branche est perçue par les gouvernements de tous pays comme un secteur d'enjeux technologiques. Elle est réputée avoir un coefficient d'innovation extrêmement élevé, et être le banc d'essai des technologies et des matériaux nouveaux - naguère l'aluminium, aujourd'hui les composites - , dont l'utilisation se diffuse ensuite dans le reste de l'industrie.

Les États ont fort logiquement essayé d'en favoriser le développement sur leur territoire. L'Allemagne ne fait pas exception, et, dans le cadre d'une coopération européenne, a accordé dès 1967 d'importantes subventions au programme d'avions civils Airbus.

On voudrait montrer ici les conséquences inattendues de cette politique industrielle. Placé en 1980 devant la nécessité d'une profonde restructuration du secteur aérospatial, restructuration dont il devient, par l'importance de son soutien financier, l'arbitre, l'État fédéral doit définir sur le vif une délicate politique d'aménagement. Les conséquences spatiales des décisions à prendre sont en effet telles que le Bund va se trouver confronté aux pouvoirs publics régionaux, les Länder, désireux de maintenir à tout prix cette activité sur leur sol. La situation va s'envenimer, dégénérer en conflit ouvert entre ces deux niveaux de la puissance publique.

L'examen des rapports de force cntrc Bund ct Länder, des contradictions entre leurs objectifs d'aménagement, de la concurrence entre Länder permet de montrer quel est l'intérêt porté en RFA aux problèmes d'aménagement industriel, et quelle peut être en période de crise la pugnacité de pouvoirs locaux acharnés à défendre leur potentiel économique et, partant, leur rang dans l'espace allemand.

\section{Une politique sectorielle coûteuse aux résultats incertains}

De 1969 à 1982, l'État fédéral a fourni sous diverses formes 3,4 milliards de marks au partenaire allemand d'Airbus Industrie. Il apparait, à l'examen des statistiques fédérales, que ces subventions constituent le seul programme public d'aide au développement industriel, par opposition aux aides à la reconversion distribuées dans les quelques autres branches subventionnées (mines, sidérurgie, construction navale), affectées par une crise structurelle (1). Pourquoi un tel engagement pour un État qui s'en tient le plus souvent à un strict libéralisme économique?

Aux yeux des hommes politiques, le maintien d'une industrie aéronautique civile en RFA et plus généralement en Europe était en jeu. Depuis les années 1950, la part de marché des Européens n'a cessé de se réduire, au profit des États-Unis. Menacés de disparition, les industriels européens cherchèrent à regrouper leurs capacités de recherche et de production, avec le soutien financier de leurs gouvernements respectifs. Le Concorde franco-britannique fut la première tentative de ce genre, mais un échec commercial retentissant (2). En 1967, les trois gouvernements français, allemand et britannique (rejoints plus tard par l'Espagne) s'engagèrent à aider un nouveau projet d'avion de ligne européen: Airbus-Industrie, alliance des constructeurs British Aerospace (GB), Aérospatiale (France), VFW et MBB (RFA), CASA (Espagne), était né.

Du côté allemand, ce soutien correspondait à des espoirs précis (3). On souhaitait conserver le potentiel technologique de pointe représenté par l'aéronautique, avec l'espoir toujours présent de voir les innovations de ce secteur se diffuser dans l'ensemble de l'industrie nationale. D'autres objectifs, plus limités, étaient d'ordre sectoriel (rééquilibrage du rapport des productions civiles et militaires de l'aéronautique allemande, autofinancement à terme du secteur).

Or les résultats n'ont, semble-t-il, pas été à la hauteur des espoirs. La percée des Airbus sur le marché mondial n'a pas vraiment permis de constater des retombées importantes pour le reste de l'industrie. Au dire de certains experts, «il faut s'abstenir d'invoquer ces effets technologiques pour justifier la politique de subventions à l'industrie aérospatiale». (4)

(1) D'après le budget fédéral.

(2) 16 exemplaires vendus, uniquement aux compagnies nationales.

(3) (1980). Basisprogramm für die deutsche Lufffahrtindustrie 1970-1974. Bonn: brochure parlementaire, imprimerie du Bundestag.

(4) Rapport HWWA (voir répertoire des sources). 
De plus, du point de vue financier, le programme Airbus n'a toujours pas permis de dégager des bénéfices, et a sécrété de nouveaux besoins, au point que l'État fédéral s'est engagé à le soutenir jusqu'en 1994 au moins, portant sa participation à 7,4 milliards de marks au total. Les difficultés d'entrée sur un marché très difficile et la conjoncture très défavorable des années 1980 expliquent en partie ces résultats (5). Il n'en reste pas moins que le Bund se trouve engagé dans une politique d'aide apparemment sans fin, d'ailleurs très mal vue par l'opinion économique allemande.

Le seul moyen d'en finir aurait été d'arrêter net le soutien. C'était un choix impossible. D'abord, le potentiel aéronautique du pays s'en serait trouvé considérablement amoindri. Or son maintien était fondamental d'un point de vue stratégique. C'est là un bonne illustration du caractère para-étatique de l'industrie aérospatiale, même si, en Allemagne, elle n'est pas nationalisée. Ensuite, l'abandon fédéral aurait signifié la suppression à court terme de tous les emplois liés à Airbus, soit environ 10000 licenciements. Comme organisme politique, le gouvernement fédéral est ainsi obligé de faire intervenir des facteurs extra-économiques. Pris au piège de son intervention, il ne peut se désengager, quel que soit le coût de celle-ci.

Une fois la solution du retrait écartée, l'État avait le devoir de réduire ces aides si discutées. Mais les industriels, parties prenantes du projet, protégés de fait des aléas du marché par les subventions, ne souhaitaient pas s'en libérer. C'est donc le gouvernement fédéral qui prit l'initiative. Il apparut très vite qu'une fusion entre les deux entreprises allemandes membres du consortium européen aboutirait à des économies significatives (6). Mais les deux firmes concernées s'y refusèrent, et les pourparlers, engagés dès 1977, furent très vite bloqués. Ce n'est qu'en 1980 que VFW et MBB, menacées d'un arrêt immédiat des subventions fédérales, furent contraintes de céder. L'État fédéral devint ainsi, par le biais des aides à Airbus, l'initiateur

(5) Même Boeing enregistra en 1981 un ticrs dc commandes en moins et licencia l'année suivante 5000 personnes.

(6) Chiffrées dans un rapport établi par la firme de conseil McKinsey à 630 millions de marks. d'une importante restructuration du secteur aérospatial. Il définit par là une véritable politique industrielle, dont l'existence si exceptionnelle pour la RFA atteste des liens organiques entre la puissance publique et la branche.

\section{Une spatialisation imprévue de la politique fédérale}

Cette vigoureuse intervention sectorielle du Bund a eu des conséquences imprévues. Les aides à Airbus ont favorisé la mise en place d'une dépendance absolue des industriels envers l'État, qui prend lui-même les initiatives majeures. Or ces événements interviennent au sein d'une industrie fortement polarisée. On distingue deux ensembles spatiaux cohérents (fig.1). À un groupe Nord où les productions civiles occupent $80 \%$ des personnels, s'oppose un ensemble méridional voué presque exclusivement aux activités militaires. Les deux pôles sont d'importance inégale: en 1980, les usines du Nord emploient 16500 personnes, celles du Sud 32 250. Ces disparités soulignent la prépondérance des activités liées à l'armement dans l'aérospatiale allemande.

Les firmes concernées par la fusion de 1980 ont de plus une indéniable personnalité régionale (fig. 2). VFW (Vereinigte Flugzeugwerke) apparaît comme une firme brêmoise: toutes ses usines sauf une, de petite taille d'ailleurs, sont situées dans la région de la Basse-Weser. Elle a dans la ville-État son siège social, son centre de recherche et sa plus grosse usine. MBB (Messerschmitt-Bölkow-Blohm), firme bavaroise à l'origine - son siège social se trouve à Ottobrunn, dans la banlieue de Munich -, est très fortement implantée dans le Sud (sept établissements en Bavière et en Bade-Wurtemberg) mais possède depuis 1969 une grosse filiale dans la région hambourgeoise (deux usines employant au total 5300 personnes) (7). Les deux entreprises se distinguent aussi par l'orientation de leur production. VFW est ainsi engagée de façon prédominante dans le secteur civil (54\% du chiffre d'affaires en 1977), tandis que MBB a bâti son succès sur l'armement.

(7) Par le rachat du constructeur HFB, issu d'une diversification des chantiers navals hambourgeois Blohm et Voss. 


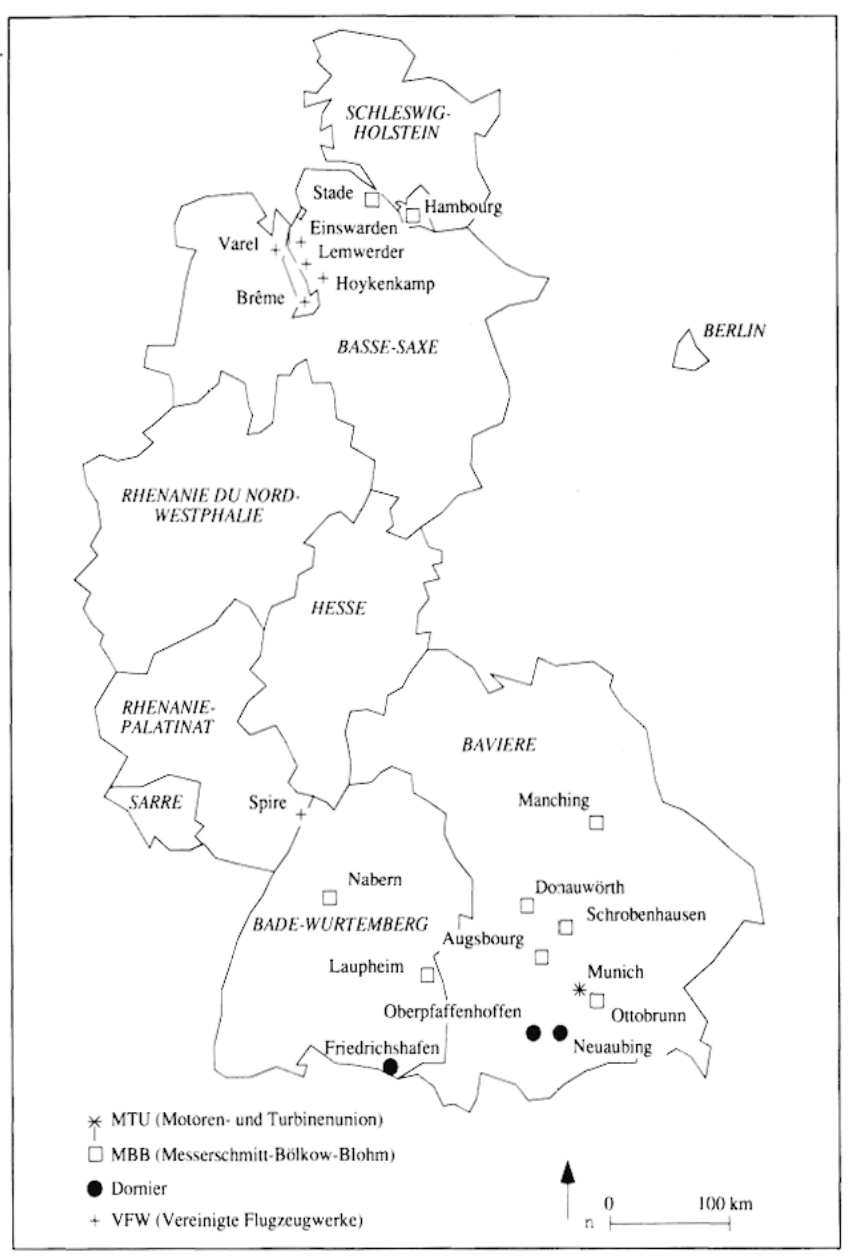

Fig. 1.- Les sites de l'industrie aérospatiale (1979).

La fusion imposée par Bonn réunit donc des partenaires très différents, dont l'un, VFW, est d'ailleurs en quasifaillite, tandis que MBB affiche d'excellents résultats. Fort logiquement, ce regroupement se traduira par une absorption de VFW par MBB.

Quelles sont les conséquences spatiales de la fusion? La nouvelle entreprise, dominée par $\mathrm{MBB}$, a son siège social à Ottobrunn. Les établissements brêmois sont donc subordonnés à un pouvoir munichois. La fusion implique aussi - et c'est dans ce but que le Bund l'a imposée - une réorganisation et un regroupement des activités des deux firmes dans certains des six domaines de production existants (fig. 2). MBB est la seule entreprise à produire des «missiles»; les 4400 personnes employées à ce travail à Manching ne sont pas touchées par le remodelage. Dans le secteur des «avions de combat», pas de modification non plus. Les 2000 salariés VFW concernés à Brême, tout comme 6000 salariés MBB répartis sur les sites d'Aug-

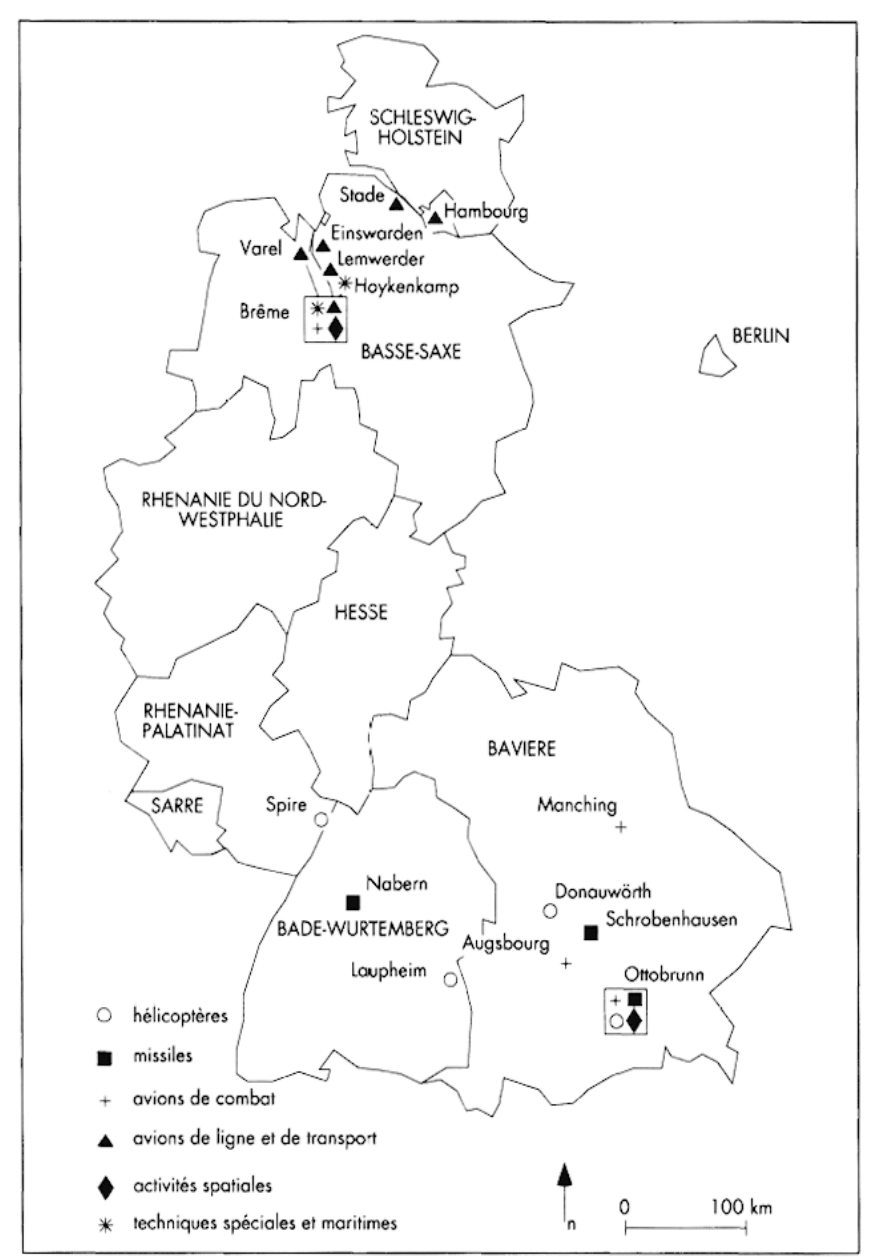

Fig. 2.- Localisation des productions de VFW et de MBB au moment de la fusion (1980).

sbourg, Manching et Ottobrunn ne sont pas menacés. Même constat pour la division «hélicoptères» (usines VFW de Spire, MBB de Donauwörth, Laupheim et Ottobrunn).

Pour les «activités spatiales» (satellites, composants de fusées), la situation est un peu différente. Elles sont florissantes des deux côtés. Leur existence n'est pas menacée, mais on pense à regrouper les équipes de recherche VFW et MBB, localisées respectivement à Brême et Ottobrunn, en un lieu unique, qui reste à choisir. Par contre, l'avenir de la division «techniques spéciales et maritimes», propre à VFW, n'est pas assuré. La nouvelle société pourrait la sacrifier, ce qui représenterait une perte de 1100 emplois répartis sur les sites de Brême et Hoykenkamp.

Ce sont en fait les activités «avions de lignc ct de transport», réductibles au seul programme Airbus, qui subissent la restructuration la plus importante. La nouvelle division, dont on veut augmenter la productivité, dispose de capacités 
de production beaucoup trop élevées, et la crise du transport aérien qui s'amorce en 1980 impose en outre à court terme une réduction de la production. On en arrive à un total de plusieurs milliers d'emplois menacés, sur les sites de Hambourg et Stade (MBB), de Brême, Einswarden, Lemwerder et Varel (VFW) (fig. 3).

La fusion a donc un effet régional sensible: c'est le Nord qui souffre. Dans ces conditions, l'État fédéral pouvait-il, après avoir forcé les industriels à se regrouper, leur laisser les mains libres pour réorganiser le nouvel ensemble? Il serait peut-être possible d'atténuer l'effet régional des restructurations par une répartition judicieuse des compressions de personnel. Mais il n'y a aucune raison de croire que, livrés à eux-mêmes, les managers bavarois maîtres du nouveau Konzern vont appliquer cette solution. On peut supposer qu'ils vont plutôt chercher à préserver l'emploi des usines de leur groupe d'origine. Or le gouvernement fédéral a tout intérêt à diriger la réorganisation. Il ne peut, au moment où il prouve qu'il est l'initiateur des principales décisions, se retirer du jeu, laisser courir le risque d'une disparition au moins partielle de l'aéronautique du paysage industriel du Nord au profit des régions méridionales, allant ainsi à l'encontre de toute politique d'aménagement du territoire basée sur le principe du rééquilibrage des activités. Le Bund ne peut causer de déséquilibre dans la structure industrielle allemande, ni laisser s'accomplir un programme massif de licenciements dans une portion très limitée de l'espace national. Bref, l'État qui subventionne ne peut s'empêcher d'être en même temps le gouvernement, c'est-à-dire l'État devant ses électeurs, et l'État fédéral, garant à ce titre des équilibres entre Länder. Comme tel, il a un devoir d'«impartialité» et ne saurait privilégier une région au profit d'une autre. Le programme sectoriel initial a donc sa traduction politique et spatiale. Le Bund est d'autant moins libre de laisser faire qu'au même moment, le Nord allemand est plongé dans une grave crise structurelle.

\section{Les États du Nord, une atmosphère de crise}

Les milliers d'emplois menacés pèsent d'autant plus lourd que les villes de Hambourg et de Brême sont confrontées toutes deux à une aggravation rapide du chômage depuis le début des années 1970, causé en partie par le déclin des activités portuaires. Cette crise de l'emploi n'est d'ailleurs

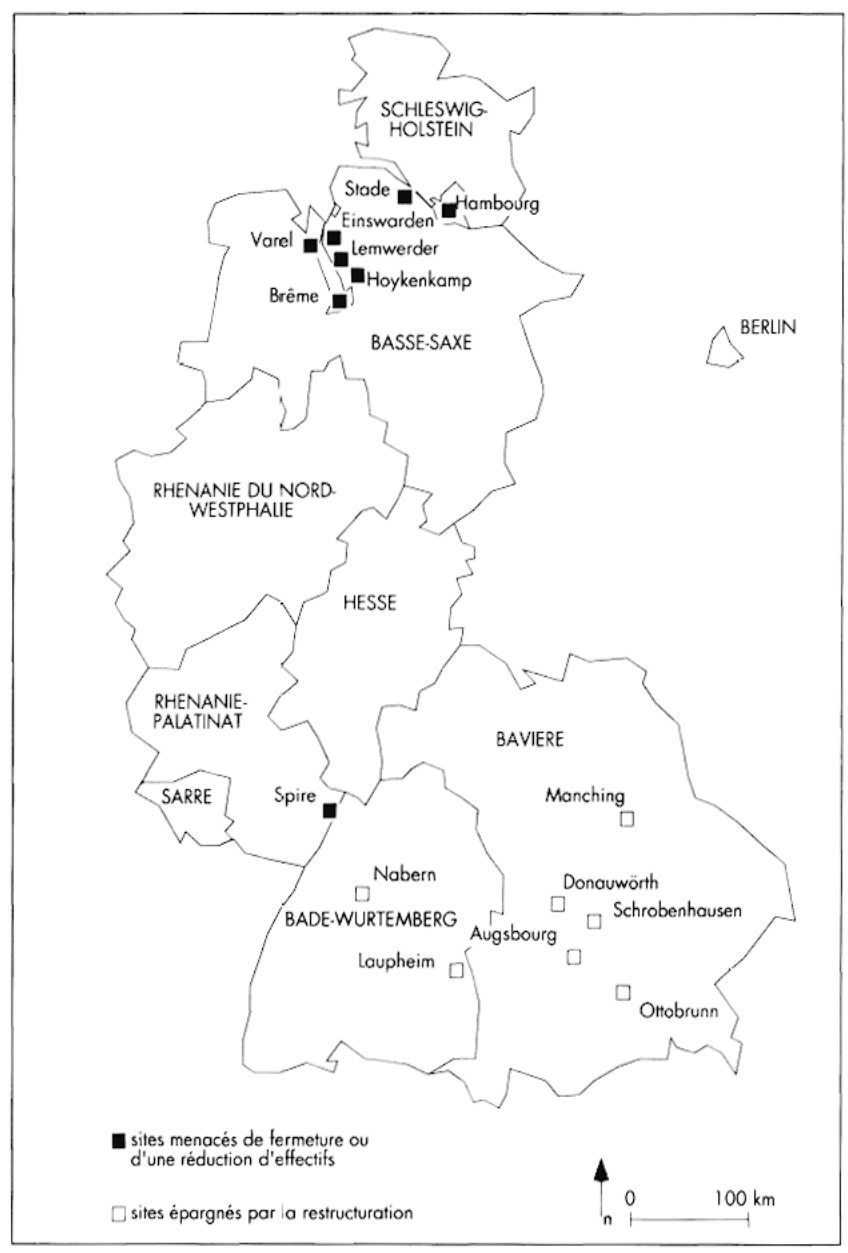

Fig. 3.-Le projet de restructuration de 1980.

que l'indice d'une crise structurelle plus grave qui affecte l'ensemble du Nord.

Région traditionnellement réputée pour son dynamisme et son taux élevé d'industrialisation, elle s'essouffle. Ainsi, dès 1975, les quatre États côtiers (Schleswig, Basse-Saxe, Hambourg et Brême) ont un taux de chômage supérieur à la moyenne fédérale. Les États du Nord, conscients de "la structure défavorable du potentiel industriel local», se rendent compte que les industries d'avenir ne s'installent guère sur leur territoire (8). Un certain malaise est présent, au point que l'on craint une véritable désindustrialisation.

(8) Collectif du Bremer Ausschuß für Wirtschaftspolitik. (1980). «Die Bedeutung der Luftfahrtindustrie im Unterweserraum». Bremer Zeitschrift für Wirtschaftspolitik, cahier 4, p.11. 
Les Länder du Sud sont au contraire les plus dynamiques; c'est en Bavière et en Bade-Wurtemberg que l'on trouve les croissances les plus fortes, dans des régions qui offrent aux industries nouvelles des espaces intacts. Ce phénomène est confirmé par les chiffres des migrations internes qui montrent depuis des années un flux persistant de migrants, notamment de diplômés, vers le Sud.

Ce «déséquilibre Nord-Sud», en allemand Süd-Nord Gefälle, est depuis plusieurs années l'objet d'une discussion et d'une polémique publiques. Il règne toutefois une regrettable confusion dans ce débat, les limites de ce «Nord» étant des plus floues, allant dans certains articles jusqu'à englober l'Allemagne moyenne (Ruhr, Sarre...). Certains économistes mettent d'ailleurs en doute l'importance réelle du phénomène de décadence industrielle du Nord, interprétant la croissance remarquable du Sud comme un rattrapage de régions peu développées à l'origine.

\section{L'intervention des Länder}

Quelle que soit la véritable interprétation, il n'en reste pas moins que la conscience d'une crise imprègne l'esprit des dirigeants du Nord. Dans cette atmosphère, les pouvoirs publics régionaux sont portés à prendre des mesures exceptionnelles pour sauver l'industrie locale, et n'assistent pas les bras croisés à la restructuration de l'un de ses fleurons: l'aérospatiale. Au moment où l'État intervient pour forcer la fusion VFW-MBB, les Länder se sont déjà depuis longtemps dotés du moyen d'influer sur les décisions qui vont ĉtrc prises. Dès 1976, le Sénat de Hambourg (exécutif régional) acquiert 20,25\% du capital de MBB. Brême se décide en 1977 à acheter 38,6\% des parts de la firme locale VFW. L'entreprise, moribonde à cette date, est sauvée par cet investissement public. Les deux Länder hanséatiques ne font que suivre l'exemple bavarois: depuis longtemps, sous l'impulsion de son ministre-président F.J. Strauß, partisan d'une politique volontariste de développement régional, la Bavière participe au capital de $\mathrm{MBB}$, pour mieux fixer sur place une industrie de haute technologie aux perspectives prometteuses.

De la crise multiforme qui frappe les métropoles du Nord, le chômage n'est qu'un des aspects. La volonté des Länder de maintenir l'activité aérospatiale sur leur sol ne se confond pas avec une simple politique de sauvegarde de l'emploi. Les pouvoirs publics régionaux n'ont d'ailleurs pas les moyens de contrecarrer l'évolution défavorable du chômage. S'ils veulent agir, ils devront se contenter d'interventions ponctuelles dans tel ou tel secteur.

Le choix de l'aérospatiale est donc significatif. Les conséquences prévisibles de sa réorganisation sont considérables. Elles sont un véritable catalogue thématique des problèmes de l'économie régionale. L'intervention des villes hanséatiques dans ce secteur témoigne de la prise de conscience de tous les problèmes structurels locaux; l'action entreprise décrit l'ensemble de la stratégie de riposte que les pouvoirs publics veulent mettre en place, elle résume et symbolise toute une politique. La décision d'intervenir activement dans l'aérospatiale paraît inséparable de l'effort fait pour penser une politique économique locale. À Brême, les pouvoirs publics commandent à un groupe d'experts locaux une étude très fouillée sur l'impact de la présence de l'aéronautique dans l'espace régional, en 1980, au moment même où se profile la restructuration (9). Il s'agit à l'évidence de disposer d'un argumentaire précis lors des pourparlers de fusion (10). Il en ressort que les autres branches industrielles locales, peu associées aux activités du secteur, n'auraient que peu à souffrir de sa disparition. L'économie régionale serait pourtant sensiblement affectée - d'autant que les salaires versés dans le secteur sont traditionnellement élevés - par la redistribution d'une masse salariale amoindrie. Les finances locales, en difficulté depuis des années (Hambourg et Brême sont alors les Länder les plus endettés de RFA), auraient à déplorer une baisse sensible de leurs revenus (taxe professionnelle, voire impôt local sur les sociétés pour Brême où VFW a son siège social).

Ces raisons financières ne sont pourtant pas prépondérantes. C'est en fait leur rang de métropole économique au sein de l'espace allemand qui est en jeu. La fusion risque de faire disparaitre des deux Länder des centres de recherche offrant un nombre élevé d'emplois très spécialisés. Les sites industriels VFW-MBB de la région pourraient être

(9) Bremer Ausschuß für Wirtschaftspolitik (1980). «Die Bedeutung der Luftfahrtindustrie im Unterweserraum». Bremer Zeitschrift für Wirtschaftspolitik, cahier 4.

(10) Hambourg dispose également de renseignements précis. 
transformés en de simples centres d'exécution, où domineraient les activités de production, plus exposés alors à des restructurations ultérieures. Or, depuis le début de la crise dans le Nord, le nombre d'entreprises entretenant des équipes de recherche a fortement diminué. Les plus gros investissements industriels de ces dernières années (l'usine Daimler-Benz à Brême en est un bon exemple) se sont effectués dans le domaine de la seule production. L'aérospatiale, avec sa proportion très élevée d'employés ayant un diplôme universitaire (35\% en moyenne, contre 7\% dans le reste de l'industrie), acquiert dans ce contexte une importance exceptionnelle. La perte de ses centres de recherche amoindrirait le patrimoine industriel, aggraverait le déficit technologique des économies locales: VFW assurait, en 1977, 47\% des dépenses de recherche effectuées dans la ville-État de Brême! C'est ainsi la possibilité pour une métropole d'offrir des prestations de services de niveau national qui est menacée.

Le maintien de ce potentiel de recherche est d'autant plus important que, face à la dégradation structurelle de leurs économies, les deux Länder avaient fortement développé le nombre de formations techniques au sein de leurs universités - rappelons que les Länder bénéficient d'une grande autonomie en matière de politique éducative ou universitaire -, en vue améliorer la qualification de la maind'œuvre locale. Il s'agit d'un effort considérable. À Hambourg, on achève alors la construction de la dernière université technique de RFA: c'est, dans un pays sans "grandes écoles», un établissement où sont formés les ingénieurs. Dans la tradition allemande des échanges avec l'industrie, cette Université doit pouvoir s'appuyer sur un tissu industriel varié et moderne pour réussir.

Il y a des deux côtés, sur la Weser comme sur l'Elbe, un lien direct et déclaré entre cette politique de formation et l'intervention dans l'aéronautique. Le maintien de celle-ci conditionne dans une certaine mesure la réussite de l'effort entrepris. En l'absence d'industries et de centres de recherche susceptibles d'accueillir les diplômés à venir, ceux-ci risquent de chercher du travail ailleurs. L'action des Länder du Nord au sein des firmes VFW et MBB s'articule à un projel cohérent pour l'économie régionale. Le maintien intégral de leur activité semble être une des conditions de réussite de la politique de régénération industrielle menée de part et d'autre. Les Länder interviennent donc vigoureuse- ment pour défendre leurs intérêts. Ils se trouvent directement confrontés à l'État fédéral. Dans ce problème de restructuration industrielle, on assiste alors à l'intervention simultanée et contradictoire de deux niveaux de la puissance publique: la question est devenue politique.

Du côté de la ville de Brême, où l'on considère qu'une absorption de VFW sous n'importe quelle forme aurait des conséquences néfastes pour le Land et consacrerait sa subordination à d'autres centres économiques, le personnel politique est irréductiblement opposé à la fusion. Depuis le début des négociations initiées par l'État fédéral (1977), les Brêmois font du blocage. Durant trois années, la ville-État, actionnaire principal de VFW, en soutient les finances chancelantes à bout de bras. Celui qui est devenu le véritable maître de l'entreprise, le sénateur à l'Économie Wilms, s'oppose directement à Bonn. On a vu que Brême ne cède finalement qu'au coup de force financier fédéral de 1980. Malgré sa remarquable détermination, sa politique a échoué, elle perd un siège social prestigieux. On peut ainsi réinterpréter en termes de rivalité régionale tous les actes du psychodrame «fusion».

Dès qu'il apparut certain que le Bund imposerait la restructuration, il eût fallu coordonner les investissements des deux firmes, surtout dans le domaine de la production d'Airbus. C'était le moment où, après une phase de lancement, le programme européen atteignait sa dimension véritablement industrielle. Il était alors nécessaire d'acquérir les presses et autres machines destinées à usiner les tôles et les plaques de composites, en nombre suffisant pour faire face à la montée en puissance de la production. Or VFW et $\mathrm{MBB}$, théoriquement associées au sein d'Airbus-Industrie, achetèrent chacune ces machines pour en équiper, l'une son usine de Stade, l'autre son site de Varel. Chacun de ces centres disposait de capacités suffisantes pour répondre aux besoins des deux entreprises. Peut-être que, dans l'espoir de voir la fusion échouer, chaque partie souhaitait attirer par ce coup de poker une part plus grande de la production d'Airbus dans ses usines.

Mais il est aussi possible que cela ait été fait délibérément dans la perspective d'une fusion prochaine, pour placer la direction future devant le fait accompli, forcer le choix du site pour le centre d'usinage nécessairement unique de la nouvelle entreprise. Ce double investissement, réalisé 
comme par hasard dans les régions économiques respectives de Hambourg et Brême, peut se comprendre alors comme une prise de gages spatiale, effectuée sous la pression des Länder actionnaires, pour assurer le maintien de l'activité de tel ou tel site, et renforcer la position d'une partie dans les négociations (fig. 1 et 2). La simultanéité des investissements annula évidemment ce calcul. Hambourg et Brême sont ainsi responsables de l'exaspération de la rivalité entre les industriels, et du gâchis financier qui en est résulté, à la fureur du bailleur de fonds du programme Airbus: Bonn.

Après l'échec des coups de force, une fois la fusion inéluctable, les Länder ne stoppent pas leurs interventions. La volonté d'en contrôler les modalités, d'en limiter les conséquences, les pousse au contraire à de nouvelles pressions, de nature strictement politique cette fois-ci, auxquelles le pouvoir fédéral ne reste pas insensible.

Ils obtiennent en effet le maintien de l'activité de tous les sites. Les sièges de division sont répartis entre les principales usines de la nouvelle entreprise. Les activités «missiles», "avions de combat» et "hélicoptères» sont dirigées depuis Ottobrunn. Mais Hambourg hérite de la direction de la branche «avions de ligne et de transport» dont les services «production» et «gestion» sont toutefois fixés à Brême. Cette ville conserve le département menacé «techniques spéciales et maritimes" et sa direction, avec les équipes de recherche afférentes.

Le même souci d'équilibre se manifeste dans la réurganisation des «activités spatiales». Les différents services de la direction divisionnaire sont répartis entre les deux sites préexistants de Brême et Ottobrunn. On évite tout déplacement massif de techniciens.

Le compromis de fusion frappe par sa complexité. Il est visiblement de nature politique. Autant que possible, on maintient les équipes de recherche là où elles se trouvent, on saupoudre les directions, donc les fonctions de commandement, entre Munich, Hambourg et Brême. C'est une indication claire de la prise en compte, dans le détail, des revendications régionales. Il faut éviter à tout prix qu'on puisse interpréter les modalités de la restructuration comme une «défaite» de telle ou telle région, et on veille en particulier à ne pas amoindrir le potentiel de recherche de chaque entité régionale. Celui-ci est en effet considéré par les $L a ̈ n d e r$ comme un indicateur pertinent de leur rang.

La contrainte politique a été particulièrement forte, obligeant les négociateurs, et singulièrement l'État fédéral, à considérer les conséquences spatiales de chacune de leurs décisions. De ce fait, le Bund aura effectué une politique d'aménagement régional, imposant à $\mathrm{MBB}$ de tempérer la brutalité d'une reprise en main selon des critères de gestion internes, dont on peut croire qu'elle n'aurait certainement pas reflété les mêmes soucis.

\section{Conclusion}

Dans cette crise et son dénouement, les politiques ont été constamment sur le devant de la scène. Länder et Bund en ont été les véritables protagonistes, effaçant les acteurs économiques privés.

Dans ces conditions, la solution trouvée était-elle viable, ou faisait-elle peser d'insupportables contraintes sur les responsables de la nouvelle firme? Les années qui ont suivi n'ayant pas apporté de modification sensible dans la division régionale du travail de l'entreprise, on en déduit que la prise en compte des intérêts régionaux ne s'est pas faite au détriment des impératifs d'une bonne gestion. Cela montre qu'une politique industrielle régionale fine a sa justification.

Par ailleurs, la sensibilité des Länder à la crise, la qualité de leur réflexion spatiale, leur volonté d'une riposte efficace au niveau local, mettent à mal le mythe du libéralisme économique allemand. Même le garant de ce mythe, l'État fédéral, pris au piège d'une aide sectorielle, a dû définir une politique sectorielle, puis n'a pu se dérober à ses conséquences spatiales. Il lui a été impossible de circonscrire son action au champ qu'il lui avait assigné: elle l'a débordé et a investi, quoi qu'il en ait, l'espace géographique.

Depuis les recherches qui ont abouti à la rédaction de cet article, la situation de l'industrie aéronautique allemande a profondément changé. Par rapport aux années 1975-1985, on observe un renversement complet de la conjoncture aéronautique mondiale. Les compagnies aériennes sont en train de renouveler leurs flottes et passent des commandes à un rythme soutenu. Ceci se répercute sur la santé d'Airbus-Industrie, et donc sur le partenaire allemand. La décision prise à la fin de 1989 d'ouvrir une 
chaîne d'assemblage Airbus à Hambourg, en sus des installations existant à Toulouse, est le signe le plus évident de la montée en puissance des capacités de production du G.I.E. Airhus en Allemagne.

La structure du secteur a aussi changé. L'absorption en 1989 de Messerschmitt (MBB) par le groupe Daimler-Benz a mis un terme au processus de concentration sectoriel entamé depuis une vingtaine d'années: Daimler dirige à présent toutes les activités aérospatiales en Allemagne.

En conséquence, un certain nombre de phénomènes décrits dans cette étude, strictement liés à l'état de la conjoncture aéronautique du début des années 1980, ont perdu de leur actualité. Les fermetures de sites, les compressions de personnel, avec toutes les conséquences sur l'emploi et la structure industrielle des régions concernées, ne sont plus à l'ordre du jour.

Ne demeure donc que ce qui, à mon sens, était le fond même de ce travail, à savoir la description du processus d'intervention de la puissance publique allemande dans un secteur industriel, avec toutes ses conséquences spatiales, et aussi ses contradictions. Ceci, au-delà de problèmes conjoncturels, permet d'analyser les rapports des différents niveaux de l'État, et les modalités de son intervention sur celui-ci, notamment en ce qui concerne l'aménagement industriel.

\section{Bibliographie ef sources}

L'industrie aérospatiale est un monde fermé, où la rétention de l'information est la règle. Les rares indications statistiques précises sur les entreprises proviennent des bilans annuels, source assez incomplète d'ailleurs. Aussi, les publications sont extrêmement rares. Faute d'ouvrage de synthèse sur le secteur, on signalera deux études:

- Collectif du Bremer Ausschuß für Wirtschaftspolitik. (1980).«Die Bedeutung der Lufffahrtindustrie im Unterweserraum». Brême: Bremer Zeitschrift für Wirtschaftspolitik, cahier 4.

- Collectif de l'Institut d'études économiques HWWA. (1980). Analyse der Subventionspolitik am Beispiel der Schiffbauindustrie sowie der Luft- und Raumfahrtindustrie. Hambourg: non publié.

En 100 planches et 500 cartes, 13 chapitres

\section{Atlas permanent de la Région-Languedoc-Roussillon}

Dans cette livraison de base, la plupart des secteurs dans les domaines sociaux, économiques, culturels, politiques de la région sont couverts.

Le traitement des données, la cartographie, la mise en pages ont été entièrement réalisés par ordinateur à la Maison de la Géographie de Montpellier.

L'Atlas existera progressivement sous forme de disquettes permettant l'affichage et certains traitements sur écran, soit en IBM PS ou PC, soit en Macintosh, grâce au Choroscope de Philippe Waniez (MGM et ORSTOM).

De nouvelles planches et des mises à jour seront proposées régulièrement.

En vente à la Maison de la Géographie, 17 rue Abbé de l'Epée, 34000 Montpellier (450 F) 